\title{
ABOUT THIS BOOK
}

Every Personal Computer (PC) you buy has to have an Operating System. This is the master program that enables the machine to carry out the routine, usually very simple, functions that make it work and enable it to run the programs you purchase. The most popular operating system is called MS-DOS. When you have finished this training course you should be able to use the important DOS commands and will then be able, should you wish to do so, to use some of the more complex and sophisticated commands that you will find in the DOS handbook that comes with every PC you buy.

\section{DESIGN OF THE LESSONS}

Each of the fourteen lessons in this book is designed to give you experience in using one or more of the DOS commands. The lessons contain a brief description of what the command does together with a step-by-step set of instructions designed to lead you easily into the use of that command (hence the title Easily into $D O S)$. These instruction sessions are displayed in a two-column format where the keys for you to press are listed in the lefthand column and the results that you will see, usually in the form of a screen display, appear in the right-hand column. Most lessons are fairly short but by the time you have worked through any one of them you should feel competent to use the commands dealt with in a real-life situation. The later lessons incorporate commands used in earlier lessons - thus enabling you to have constant revision.

The course book, as you will have noticed, is accompanied by a floppy disk. This is your practice disk and you will find that you will be using it constantly. It is a good idea for you to copy the disk and preserve the original. You can then work on the copy, safe in the knowledge that if you do any damage to the disk all is not lost.

We hope that you find the course interesting, stimulating and above all useful. In fact, a computer can be fun even if you do not play games on it! 